\title{
Thermally induced 0- $\pi$ phase transition in Josephson junctions through a ferromagnetic oxide film
}

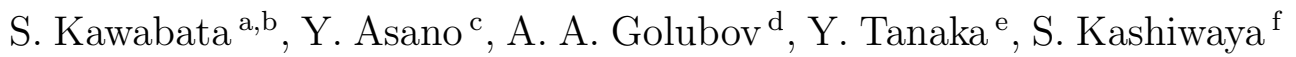 \\ ${ }^{a}$ Nanosystem Research Institute (NRI), National Institute of Advanced Industrial Science and Technology (AIST), Tsukuba, \\ Ibaraki 305-8568, Japan \\ b CREST, Japan Science and Technology Corporation (JST), Kawaguchi, Saitama 332-0012, Japan \\ ${ }^{\mathrm{c}}$ Department of Applied Physics, Hokkaido University, Sapporo, 060-8628, Japan \\ ${ }^{\mathrm{d}}$ Faculty of Science and Technology, University of Twente, P.O. Box 217, 7500 AE Enschede, The Netherlands \\ e Department of Applied Physics, Nagoya University, Nagoya, 464-8603, Japan \\ ${ }^{\mathrm{f}}$ Nanoelectronics Research Institute (NeRI), AIST, Tsukuba, Ibaraki 305-8568, Japan
}

\begin{abstract}
We investigate the Josephson transport through a ferromagnetic oxide film, e.g., $\mathrm{La}_{2} \mathrm{BaCuO}_{5}$, theoretically. Using the recursive Green's function technique, we found the formation of a $\pi$-junction in such systems. Moreover the $0-\pi$ phase transition is induced by increasing the temperature. Such ferromagnetic-oxide based Josephson junctions may become an element in the architecture of future quantum computers.
\end{abstract}

Key words: Josephson junction, Spointronics, Ferromagnetic oxide, Quantum computer, Green's function method PACS: 74.50.+r, 03.65.Yz, 05.30.-d

\section{Introduction}

It is well established that a superconducting phase difference of $\pi$ can be arranged between two $s$-wave superconductors (Ss) when separating them by a suitably chosen ferromagnetic metals (FMs) $[1,2]$. Transitions between the $\pi$-state and the 0 -state of such S/FM/S Josephson junctions have been revealed in experiments through oscillations of the Josephson critical current $I_{c}$ with varying thickness of the FM [3] or with varying temperature [4]. The $\pi$ Josephson junction is currently of considerable interest as an element complementary to the usual Josephson junction in the development of functional nano-structures including superconducting electronics [5].

Recently, quiet qubits consisting of a S/FM/S $\pi$ junction have been theoretically proposed $[6,7]$. In the quiet qubits, a quantum two level system is spon- taneously generated and therefore it is expected to be robust to the decoherence by the fluctuation of the external magnetic field. From the viewpoint of the quantum decoherence, however, S/FM/S junctions is inherently identical with $\mathrm{S} / \mathrm{NM} / \mathrm{S}$ junctions, where NM is a nonmagnetic normal-metal. Therefore a gapless quasiparticle excitation in the FM region is inevitable. This feature gives a strong Ohmic dissipation [8] and the coherence time of S/FM/S quiet qubits is bound to be very short. Thus the realization of the $\pi$ junction without using such metallic ferromagnets are highly desired for qubit applications [9-12].

On the other hand, recently, we have developed a numerical method to calculate the Josephson current by taking into account the band structure of ferromagnetic materials based on the Recursive Green's function method [13-15]. By use of this method, we numerically found the formation 
of the $\pi$-state for the $s$-wave junction through a ferromagnetic oxide (FOs) which is a kind of ferromagnetic insulators. Moreover we have found that the $0-\pi$ transition is induced by increasing the thickness of the oxide layer [14-16]. Heretofore, however, we have only considered the Josephson transport at the very low temperature regime, i.e., $T \ll T_{c}$, where $T_{c}$ is the superconductor transition temperature. In this paper we will investigate the finite-temperature Josephson transport and show that the $0-\pi$ phase transition can be realized by increasing the temperature.

\section{Electronic state of ferromagnetic oxides}

The typical DOS of FOs for each spin direction is shown schematically in Fig. 1. One of the representative material of such magnetic materials is half-filled $\mathrm{La}_{2} \mathrm{BaCuO}_{5}$ (LBCO) [17-19]. The exchange splitting $V_{\text {ex }}$ is estimated to be $0.34 \mathrm{eV}$ by a first-principle band calculation using the spin-polarized local density approximation [20]. Since the exchange splitting $V_{\text {ex }}$ is large and the bands are originally half-filled, the system becomes the ferromagnetic insulator. In

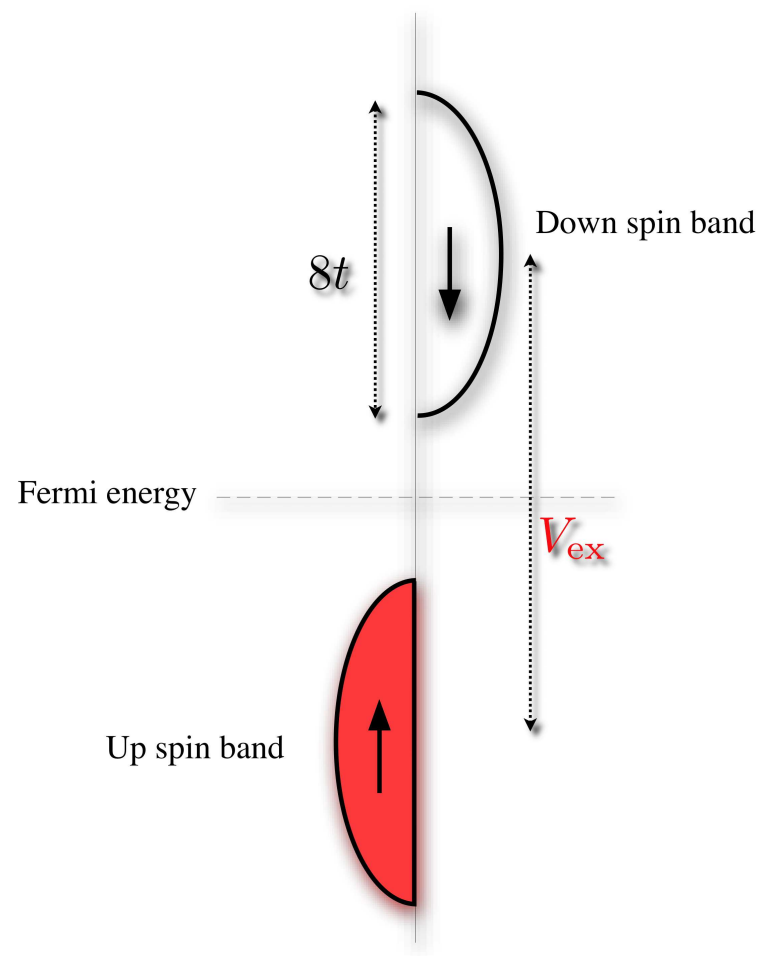

Fig. 1. (Color online) The density of states for each spin direction for a ferromagnetic oxide, e.g., $\mathrm{La}_{2} \mathrm{BaCuO}_{5}$. the next section, we calculate the Josephson current through such oxide films numerically.

\section{Numerical calculation of Josephson current}

In this section, we outline a numerical calculation method for the Josephson current of S/FO/S junctions based on the recursive Green's function technique [21]. Let us consider a two-dimensional square tight-binding lattice for the $\mathrm{S} / \mathrm{FO} / \mathrm{S}$ junction. The vector $\boldsymbol{r}=j \boldsymbol{x}+m \boldsymbol{y}$ points to a lattice site, where $\boldsymbol{x}$ and $\boldsymbol{y}$ are unit vectors in the $x$ and $y$ directions, respectively. In the $y$ direction, we apply the periodic boundary condition for the number of lattice sites being $W$.

Electronic states in a superconductor are described by the mean-field Hamiltonian

$$
\begin{aligned}
H_{\mathrm{BCS}} & =\frac{1}{2} \sum_{\boldsymbol{r}, \boldsymbol{r}^{\prime} \in \mathrm{S}}\left(\tilde{c}_{\boldsymbol{r}}^{\dagger} \hat{h}_{\boldsymbol{r}, \boldsymbol{r}^{\prime}} \tilde{c}_{\boldsymbol{r}^{\prime}}-\overline{\tilde{c}}_{\boldsymbol{r}} \hat{h}_{\boldsymbol{r}, \boldsymbol{r}^{\prime}}^{*} \overline{\tilde{c}_{\boldsymbol{r}^{\prime}}^{\dagger}}\right) \\
& +\frac{1}{2} \sum_{\boldsymbol{r} \in \mathrm{S}}\left(\tilde{c}_{\boldsymbol{r}}^{\dagger} \hat{\Delta} \overline{\tilde{c}_{\boldsymbol{r}}^{\dagger}}-\overline{\tilde{c}_{\boldsymbol{r}}} \hat{\Delta}^{*} \tilde{c}_{\boldsymbol{r}}\right) .
\end{aligned}
$$

In this equation,

$\hat{h}_{\boldsymbol{r}, \boldsymbol{r}^{\prime}}=\left\{-t_{s} \delta_{\left|\boldsymbol{r}-\boldsymbol{r}^{\prime}\right|, 1}+\left(-\mu_{s}+4 t_{s}\right) \delta_{\boldsymbol{r}, \boldsymbol{r}^{\prime}}\right\} \hat{\sigma}_{0}$,

with $\overline{\tilde{c}}_{\boldsymbol{r}}=\left(c_{\boldsymbol{r}, \uparrow}, c_{\boldsymbol{r}, \downarrow}\right)$, where $c_{\boldsymbol{r}, \sigma}^{\dagger}\left(c_{\boldsymbol{r}, \sigma}\right)$ is the creation (annihilation) operator of an electron at $\boldsymbol{r}$ with spin $\sigma=(\uparrow$ or $\downarrow), \overline{\tilde{c}}$ means the transpose of $\tilde{c}$, and $\hat{\sigma}_{0}$ is $2 \times 2$ unit matrix. The chemical potential $\mu_{s}$ is set to be $2 t_{s}$ for superconductors. In superconductors, the hopping integral $t_{s}$ is considered among nearest neighbor sites and we choose

$\hat{\Delta}=i \Delta \hat{\sigma}_{y}$

where $\Delta$ is the amplitude of the pair potential in the $s$-wave symmetry channel, and $\hat{\sigma}_{y}$ is a Pauli matrix.

The Hamiltonian of the ferromagnetic oxide film is given by a single-band tight-binding model as

$$
\begin{aligned}
H_{\mathrm{FO}} & =-t \sum_{\boldsymbol{r}, \boldsymbol{r}^{\prime}, \sigma} c_{\boldsymbol{r}, \sigma}^{\dagger} c_{\boldsymbol{r}^{\prime}, \sigma}-\sum_{\boldsymbol{r}}(4 t-\mu) c_{\boldsymbol{r}, \uparrow}^{\dagger} c_{\boldsymbol{r}, \uparrow} \\
& +\sum_{\boldsymbol{r}}\left(4 t-\mu+V_{\mathrm{ex}}\right) c_{\boldsymbol{r}, \downarrow}^{\dagger} c_{\boldsymbol{r}, \downarrow},
\end{aligned}
$$

where $V_{\text {ex }}$ is the exchange splitting between the up and down spin band. When $V_{\mathrm{ex}}>8 t$, this Hamiltonian describes the ferromagnetic oxide as shown in Fig. 1. The chemical potential $\mu$ is set to be 
$V_{\text {ex }} / 2+4 t$. A superconductor and a ferromagnetic oxide are connected by

$$
\begin{aligned}
H_{c 1}= & -t_{s} \sum_{m, \sigma}\left(c_{0, m, \sigma}^{\dagger} c_{1, m, \sigma}+c_{L_{F}, m, \sigma}^{\dagger} c_{L_{F}+1, m, \sigma}\right. \\
& + \text { h.c. }),
\end{aligned}
$$

where $L_{F}$ is the thickness of the FO layer.

The Hamiltonian is diagonalized by the Bogoliubov transformation and the Bogoliubov-de Gennes equation is numerically solved by the recursive Green function method[21]. We calculate the Matsubara Green function in a ferromagnetic oxide,

$$
\check{G}_{\omega_{n}}\left(\boldsymbol{r}, \boldsymbol{r}^{\prime}\right)=\left(\begin{array}{cc}
\hat{g}_{\omega_{n}}\left(\boldsymbol{r}, \boldsymbol{r}^{\prime}\right) & \hat{f}_{\omega_{n}}\left(\boldsymbol{r}, \boldsymbol{r}^{\prime}\right) \\
-\hat{f}_{\omega_{n}}^{*}\left(\boldsymbol{r}, \boldsymbol{r}^{\prime}\right) & -\hat{g}_{\omega_{n}}^{*}\left(\boldsymbol{r}, \boldsymbol{r}^{\prime}\right)
\end{array}\right),
$$

where $\omega_{n}=(2 n+1) \pi T$ is the Matsubara frequency, $n$ is an integer number, and $T$ is a temperature. The finite temperature Josephson current is given by

$I_{J}(\phi)=-i e t T \sum_{\omega_{n}} \sum_{m=1}^{W} \operatorname{Tr}\left[\check{G}_{\omega_{n}}\left(\boldsymbol{r}^{\prime}, \boldsymbol{r}\right)-\check{G}_{\omega_{n}}\left(\boldsymbol{r}, \boldsymbol{r}^{\prime}\right)\right]$,

with $\boldsymbol{r}^{\prime}=\boldsymbol{r}+\boldsymbol{x}$. Note that the Green function in Eq. (6) is a $4 \times 4$ matrix representing spin and Nambu spaces. Throughout this paper we fix the following parameters: $W=5$, and $\Delta_{0}=0.01 t$, and assume $t=t_{s}$ for simplicity.

\section{Result}

The $0-\pi$ phase diagram for $T=0.01 T_{c}$ depending on the exchange splitting $V_{\mathrm{ex}}$ and the thickness of FO layer $L_{F}$ is shown in Fig. 2. The black (white) regime corresponds to the $\pi$ - $(0-)$ junction, i.e., $I_{J}=-(+) I_{C} \sin \phi$. We find that the atomicscale $0-\pi$ phase transition is induced by increasing the thickness of FO layer $[14,15]$.

Next we consider the finite-temperature Josephson transport. The temperature dependence of $I_{c}$ for the odd $L_{F}$ junction is plotted in Fig. 3 . In the case of the tunneling limit $\left(V_{\mathrm{ex}} \gg t\right),\left|I_{c}\right|$ obeys the well-known Ambegaokar-Baratoff formula. By decreasing the exchange splitting $V_{\mathrm{ex}}$, we found the appearance of an anomalous thermally-induced $0-\pi$ transition for the $\mathrm{S} / \mathrm{FO} / \mathrm{S}$ junction. More detailed discussion for above peculiar results will be given elsewhere.

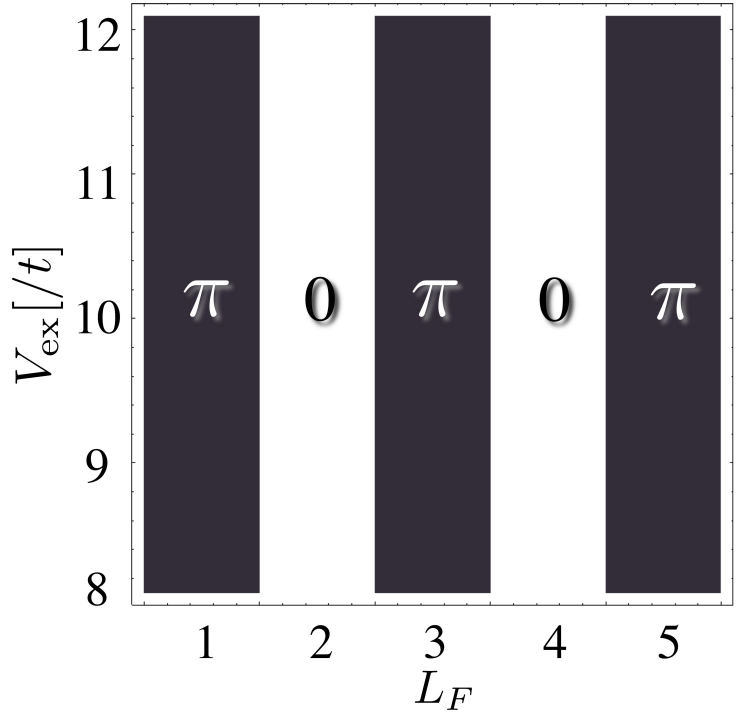

Fig. 2. The phase diagram depending on the exchange splitting $V_{\text {ex }}$ and the thickness of the ferromagnetic oxide (FO) layer $L_{F}$ for the $\mathrm{S} / \mathrm{FO} / \mathrm{S}$ Josephson junction at $T=0.01 T_{c}$. The black and white regime correspond to the $\pi$ and 0 junction, respectively.

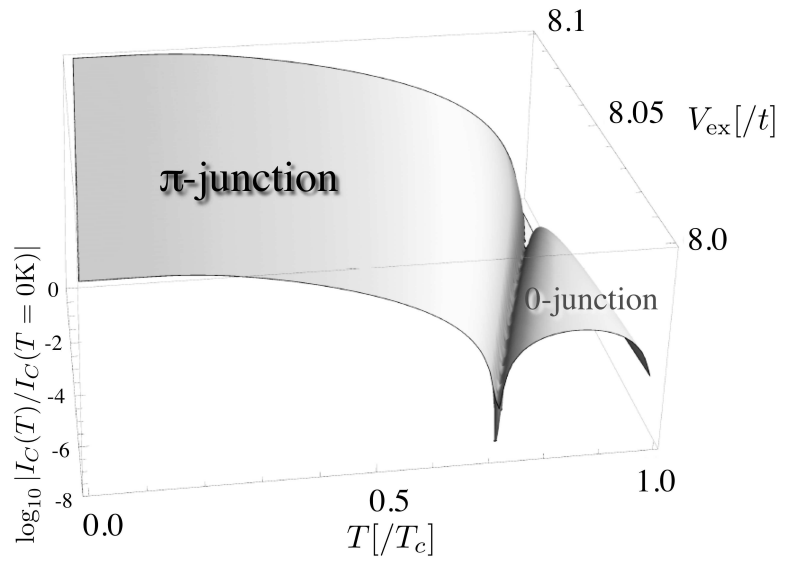

Fig. 3. The Josephson critical current $I_{C}$ as a function of the temperature $T$ and the exchange splitting $V_{\mathrm{ex}}$ for the $\mathrm{S} / \mathrm{FO} / \mathrm{S}$ Josephson junction with $L_{F}=3$.

\section{Summary}

To summarize, we have studied the finitetemperature Josephson transport in S/ferromagneticoxide/S junction by use of the recursive Green's function method. We found that the $0-\pi$ transition can be induced by increasing the temperature. Therefore we can experimentally confirm the $\pi$ junction behavior by measuring the temperature dependence of the Josephson critical current. Such FO junctions may become an element in the archi- 
tecture of $\pi$-junction based qubits [9-12].

\section{Acknowledgements}

This work was supported by CREST-JST, and a Grant-in-Aid for Scientific Research from the Ministry of Education, Science, Sports and Culture of Japan (Grant No. 22710096).

\section{References}

[1] A. I. Buzdin, Rev. Mod. Phys. 77 (2005) 935.

[2] A. A. Golubov, M. Y. Kupriyanov, E. Il'ichev, Rev. Mod. Phys. 76 (2004) 411.

[3] T. Kontos, M. Aprili, J. Lesueur, F. Genêt, B. Stephanidis, R. Boursier, Phys. Rev. Lett. 89 (2002) 137007.

[4] V. V. Ryazanov, V. A. Oboznov, A. Y. Rusanov, A. V. Veretennikov, A. A. Golubov, J. Aarts, Phys. Rev. Lett. 86 (2001) 2427.

[5] T. Ortlepp, Ariando, O. Mielke, C. J. M. Verwijs, K. F. K. Foo, H. Rogalla, F. H. Uhlmann, H. Hilgenkamp, Science 312 (2006) 1495.

[6] L. B. Ioffe, V. B. Geshkenbein, M. V. Feigel'man, A. L. Fauchére, G. Blatter, Nature 398 (1999) 679.

[7] G. Blatter, V. B. Geshkenbein L. B. Ioffe, Phys. Rev. B 63 (2001) 174511.

[8] A. D. Zaikin, S. V. Panyukov, Sov. Phys. JETP 62 (1985) 137.

[9] S. Kawabata, S. Kashiwaya, Y. Asano, Y. Tanaka, Physica C 437-438 (2006) 136.

[10] S. Kawabata, S. Kashiwaya, Y. Asano, Y. Tanaka, A. A. Golubov, Phys. Rev. B 74 (2006) 180502(R).

[11] S. Kawabata, A. A. Golubov, Physica E 40 (2007) 386.

[12] S. Kawabata, Y. Asano, Y. Tanaka, S. Kashiwaya, A. A. Golubov, Physica C 468 (2008) 701.

[13] S. Kawabata, Y. Asano, Y. Tanaka, S. Kashiwaya, Physica C 469 (2009) 1621.

[14] S. Kawabata, Y. Asano, Int. J. Mod. Phys. B 23 (2009) 4320.

[15] S. Kawabata, Y. Asano, Y. Tanaka, S. Kashiwaya, Physica E 42 (2010) 1010.

[16] S. Kawabata, Y. Asano, Y. Tanaka, A. A. Golubov, S. Kashiwaya, Phys. Rev. Lett. 104 (2010) 117002.

[17] F. Mizuno, H. Masuda, I. Hirabayashi, S. Tanaka, M. Hasegawa, U. Mizutani, Nature 345 (1990) 788.

[18] H. Masuda, F. Mizuno, I. Hirabayashi, S. Tanaka, Phys. Rev. B 43 (1991) 7881.

[19] W. Ku, H. Rosner, W. E, Pickett, R. T. Scalettar, Phys. Rev. Lett. 89 (2002) 167204.

[20] V. Eyert, K. H. Höc, P. S. Riseborough, Europhys. Lett. 31 (1995) 385.

[21] Y. Asano, Phys. Rev. B 63 (2001) 052512. 